\title{
SOME SUBGROUPS OF THE THOMPSON GROUP
}

\author{
ROBERT A. WILSON \\ (Received 18 September 1985) \\ Communicated by $\mathrm{H}$. Lausch
}

\begin{abstract}
We determine all conjugacy classes of maximal local subgroups of Thompson's sporadic simple group, and all maximal non-local subgroups except those with socle isomorphic to one of five particular small simple groups.
\end{abstract}

1980 Mathematics subject classification (Amer. Math. Soc.): 20 D 08.

\section{Introduction}

In this paper we classify all the maximal $p$-local subgroups of Thompson's simple group $T h$ of order $90,745,943,887,872,000=2^{15} \cdot 3^{10} \cdot 5^{3} \cdot 7^{2} \cdot 13 \cdot 19.31$, and also partially classify the non-local subgroups. The existence of this group was originally conjectured by $\mathrm{J}$. G. Thompson as a subgroup of the then unconstructed Monster group, in which the $3 C$-centralizer is $3 \times T h$, and was first constructed by P. E. Smith and J. G. Thompson (see [2]) as a group of real $248 \times 248$ matrices.

Our main result is the following theorem.

THEOREM. Any maximal subgroup of Th is either (A) a conjugate of one of the maximal subgroups given in Table 1 . or (B) a conjugate of a particular group $L_{2}(19): 2$ if $L_{2}(19)$ is a subgroup of $T h$

() 1988 Australian Mathematical Society $0263-6115 / 88 \$ A 2.00+0.00$ 
or (C) the normalizer of a simple group $S$ with trivial centralizer, where $S \cong A_{6}$, $L_{2}(7), L_{3}(3)$ or $U_{3}(3)$.

$$
\begin{array}{ll}
2_{+}^{1+8} \cdot A_{9} & 5^{2}: G L_{2}(5) \\
2^{5} \cdot L_{5}(2) & 7^{2}:\left(3 \times 2 S_{4}\right) \\
\left(3 \times G_{2}(3)\right): 2 & 31: 15 \\
\left(3^{3} \times 3_{+}^{1+2}\right) \cdot 3_{+}^{1+2}: 2 S_{4} & { }^{3} D_{4}(2): 3 \\
3^{2} \cdot\left[3^{7}\right] .2 S_{4} & U_{3}(8): 6 \\
\left(3 \times 3^{4}: 2 \cdot A_{6}\right): 2 & M_{10} \\
5_{+}^{1+2}: 4 S_{4} & S_{5}
\end{array}
$$

TABLE 1

REMARK. Some further restrictions on possible subgroups of type (C) are given in the final section of the paper.

Note. Our notation for groups, conjugacy classes, characters, etc. follows the ATLAS [1].

Note added in proof. S. Linton has now shown that $L_{2}(19)$ is a subgroup of $T h$, and that the cases $S \cong A_{6}, L_{2}(7)$ and $U_{3}(3)$ do not arise in part $(C)$ of the Theorem.

\section{The 2-local subgroups}

There is just one class of involutions in the Thompson group, with centralizer $2_{+}^{1+8} \cdot A_{9}$. In this group, the action of the $A_{9}$ on the $2^{8}$ is not the deleted permutation representation, but may be obtained from the latter by applying the triality automorphism of $O_{8}^{+}(2)$. All the non-central involutions in $2^{1+8}$ are conjugate under the action of $A_{9}$, so we obtain one class of four-groups with centralizer $2^{2} \cdot\left[2^{9}\right] \cdot L_{3}(2)$. Now the involutions of cycle shape $\left(2^{2} 1^{5}\right)$ in $A_{9}$ do not lift to involutions in $T h$. An involution of cycle shape $\left(2^{4} 1\right)$ has centralizer $2^{3} S_{4}$ in $A_{9}$, and its fixed space in the $2^{8}$ has order $2^{4}$. Hence the stabilizer of a corresponding four-group in $2^{1+8} A_{9}$ has order at most $2^{11} \cdot 3$. On the other hand, the structure constant $\xi(2 A, 2 A, 2 A)=1 / 2^{14} .3 .7+1 / 2^{10} .3$, so we have proved the following lemma. 
LEMMA 2.1. There are just two classes of four-groups in Th, one with centralizer of order $2^{14} .3 .7$, and the other with centralizer of order $2^{10} .3$.

Using the geometry of $O_{8}^{+}(2)$, we can find the orbits of $A_{9}$ on the totally isotropic subspaces of the $2^{8}$. (The easiest way to do this is to work in the deleted permutation representation, and then apply the triality automorphism, so that 1-spaces become 4-spaces and so on.) The orbits are as follows:

$\begin{array}{ll}\text { Orbit size(s) } & \\ 135 & \text { on the } 135 \text { points (1-spaces) } \\ 315+1260 & \text { on the } 1575 \text { lines (2-spaces) } \\ 135+1800 & \text { on the } 2025 \text { 3-spaces } \\ 9+126 & \text { on the } 1354 \text {-spaces of the first kind } \\ 135 & \text { on the } 1354 \text {-spaces of the second kind }\end{array}$

We define any isotropic space to be nice if it is contained in one of the 4-spaces in the orbit of size 9. The 9 nice 4-spaces are disjoint, and each contains 15 1-spaces, 35 2-spaces and 15 3-spaces, so by counting we see that any nice space is contained in a unique nice 4-space. Furthermore, a subspace is nice if and only if all its 2-dimensional subspaces are nice.

The normalizer of a four-group of the first type is $N\left(2 A^{2}\right)_{1} \cong 2^{2} \cdot\left[2^{9}\right] \cdot\left(S_{3} \times\right.$ $\left.L_{3}(2)\right)$, in which the $L_{3}(2)$ acts on the $\left[2^{9}\right]$ as one copy of the natural representation together with two copies of its dual. Furthermore, the natural module is a submodule, so gives rise to a normal subgroup $2^{5}$ in $N\left(2 A^{2}\right)_{1}$. This $2^{5}$-group corresponds to a nice 4-space in the $2^{8}$, so its normalizer contains both $2^{5} .2^{4} . A_{8}$ and $2^{5} .2^{6} .\left(L_{3}(2) \times S_{3}\right)$, and therefore has the shape $2^{5} . L_{5}(2)$. All four-groups in this $2^{5}$-group are of the first type, and correspond to the nice 2 -spaces in $2^{1+8}$. Hence the isotropic 2-spaces in the 1260-orbit are conjugate to the second type of four-group. The normalizer of this latter four-group is $N\left(2 A^{2}\right)_{2} \cong\left(2^{2} \times 2^{1+4}\right)$. $\left(S_{4} \times S_{3}\right)<2^{1+8} A_{9}$, in which the $S_{4} \times S_{3}$ acts on $6+3$ letters in the $A_{9}$-image. Indeed, the four-group centralizer is $\left(2^{2} \times 2^{1+4}\right) \cdot S_{4}$, in which the $S_{4}$ fixes 3 letters, since only $3 A$-elements centralize isotropic 2 -spaces. Hence all involutions in the $S_{4}$ have cycle type $\left(2^{2} 1^{5}\right)$ in the $A_{9}$, so do not lift to involutions in $T h$. Hence any elementary Abelian 2-group not in the nice $2^{5}$ is in a unique group $2^{1+8}$, and so its normalizer is in $2^{1+8} A_{9}$. This concludes the proof of

THEOREM 2.2. Any 2-local subgroup of Th is contained in either $N(2 A) \cong$ $2^{1+8} \cdot A_{9}$ or $N\left(2 A^{5}\right) \cong 2^{5} \cdot L_{5}(2)$. 


\section{The $p$-local subgroups, $p \geqslant 5$}

For each prime $p \geqslant 5$ dividing the order of the Thompson group, there is a unique class of subgroups of order $p$, and their normalizers are as follows:

$$
\begin{aligned}
& N(5 A) \cong 5_{+}^{1+2}: 4 S_{4}, \\
& N(7 A) \cong\left(7: 3 \times L_{2}(7)\right): 2<{ }^{3} D_{4}(2): 3, \\
& N(13 A) \cong(13: 6 \times 3) \cdot 2<\left(3 \times G_{2}(3)\right): 2, \\
& N(19 A) \cong 19: 18<U_{3}(8): 6, \\
& N(31 A B) \cong 31: 15 .
\end{aligned}
$$

The groups $N(5 A)$ and $N(31 A B)$ will turn out to be maximal subgroups of $T h$.

There is a unique class of groups of each of the orders $5^{2}$ and $7^{2}$, and so their normalizers are

$$
\begin{aligned}
& N\left(5 A^{2}\right) \cong 5^{2}: G L_{2}(5), \\
& N\left(7 A^{2}\right) \cong 7^{2}:\left(3 \times 2 S_{4}\right)
\end{aligned}
$$

both of which are maximal subgroups of $T h$.

\section{The 3-local subgroups}

There are three classes of elements of order 3 in $T h$, with normalizers

$$
\begin{aligned}
& N(3 A) \cong\left(3 \times G_{2}(3)\right): 2, \\
& N(3 B) \cong\left(3^{3} \times 3_{+}^{1+2}\right) \cdot 3_{+}^{1+2}: 2 S_{4}, \\
& N(3 C) \cong\left(3 \times 3^{4}: 2 A_{6}\right): 2 .
\end{aligned}
$$

For a proof that $N(3 B)$ has the above structure, and for further details, see below.

Now by looking at the character value on involutions, we see that the 248-character of $T h$ restricts to $3 \times G_{2}(3)$ as $1 \otimes(1+91)+(\omega+\bar{\omega}) \otimes 78$, where the characters of the group of order 3 are denoted by their values on a generator, and those of $G_{2}(3)$ by their degrees. Hence we have the following class fusion

$\begin{array}{lllll}G_{2}(3) \text {-class } & 3 A / B & 3 C & 3 D & 3 E \\ \text { Th-class } & 3 B & 3 B & 3 C & 3 A \\ \text { diagonal elements } & 3 A & 3 A & 3 B & 3 C \\ 3^{2} \text {-type } & 3 A_{3} B_{1} & 3 A_{3} B_{1} & 3 A_{1} B_{2} C_{1} & 3 A_{2} C_{2} \\ 3^{2} \text {-centralizer } & {\left[3^{6}\right]: 2 A_{4}} & {\left[3^{7}\right]} & 3^{5}: 2 & 3^{5}: 2\end{array}$

Now let $Y$ be an elementary Abelian 3-group generated by $3 A$-elements. If every pair of $3 A$-elements in $Y$ generates a group of type $3 A_{3} B_{1}$, then $Y$ contains a unique $3 B$-pure subgroup of index 3 , and so $N(Y)$ is contained in the normalizer 
of a $3 B$-pure group. Otherwise, $Y$ contains a group of type $3 A_{1} B_{2} C_{1}$ or $3 A_{2} C_{2}$, each of which has centralizer of shape $3^{5}: 2$. Hence $C(Y)$ contains a unique Sylow 3 -subgroup, which in each case is an elementary Abelian group of order $3^{5}$, whose normalizer we will find later. In fact we will see that these two $3^{5}$-groups are conjugate in $T h$.

Next we consider the case of an elementary Abelian group $Y$ generated by $3 C$-elements, and containing no $3 A$-elements. We first need to study the $3 C$-normalizer in some detail. We have $C(3 C) \cong 3 \times 3^{4}: S L_{2}(9)$, in which the group $S L_{2}(9) \cong 2 A_{6}$ acts naturally on $3^{4} \cong\left(\mathbf{F}_{9}\right)^{2}$, where $\mathbf{F}_{9}$ is the field $\{0, \pm 1, \pm i$, $\pm 1 \pm i$ \} of order 9 .

The 243 linear representations of the normal $3^{5}$-subgroup $E$ are fused by $2 \dot{A}_{6}$ to give representations of degrees 1 and 80 , which we denote by $1 a, 1 b, 1 c, 80 a$, $80 b, 80 c$. Furthermore the outer elements of $2 S_{6}$ fuse $1 b$ to $1 c$ and $80 b$ to $80 c$. Hence from the character value on the $3 C$-element we see that the 248-character of $T h$ restricts to $3^{5}: 2 S_{6}$ as $1 b^{4} c^{4}+80 a b c$, that is, four copies of each of the non-trivial linear characters, plus one copy of each of the 80-dimensional characters. This shows that $E \cong 3^{5}$ has type $3 C_{1} B_{40} C_{80}$, and $N(E)$ is not transitive on the $3 C$-elements in $E$, since the order of the Thompson group is not divisible by $3^{11}$.

Now consider the subgroup $3 \times S L_{2}(9) \cong 3 \times 2 A_{6}$. Since we have already seen $3^{2}$-groups of types $3 A_{1} B_{2} C_{1}$ and $3 A_{2} C_{2}$ in the involution centralizer, it follows that both of these are represented in $3 \times 2 A_{6}$. Furthermore, since the Sylow 3-subgroup of $S L_{2}(9)$ fixes a 1-space in the natural representation over $\mathbf{F}_{9}$, we see that there is a group $F \cong 3^{5}$ containing both these $3^{2}$-groups.

Now the Sylow 3-group in $\left(\mathbf{F}_{9}\right)^{2}: S L_{2}(9)$ is a group $3^{2+4}$ in which each non-central element has order 3 and centralizer $3^{4}$. There are 10 such groups $3^{4}$, of which one is the vector space $\left(\mathbf{F}_{9}\right)^{2}$ and another contains elements of the complementary $S L_{2}(9)$. The remaining 8 groups are permuted transitively by the Sylow 3-normalizer $3^{2+4}:\left[2^{4}\right]$ in $3^{4}: 2 S_{6}$. Hence these also give rise to $3^{5}$-groups of type $3 B_{40} C_{81}$, conjugate to $E$ but seen from the point of view of one of the $3 C$-elements in the 80-orbit. This determines the conjugacy classes of all elements of order 3 in $N(3 C)$, and in particular shows that the $3^{5}$-group $F$ defined above has type $3 A_{54} B_{40} C_{27}$, and that any two commuting $3 C$-elements generate a $3^{2}$-group of type $3 A_{2} C_{2}$ or $3 B_{1} C_{3}$.

Now any elementary Abelian group generated by $3 C$-elements is either in a conjugate of $E$, in which case it contains a unique $3 B$-pure subgroup of index 3 , or else its centralizer has a unique Sylow 3-group, which is conjugate to $F$. Now $F$ contains $3 A_{2} C_{2}$-subgroups, so is conjugate to both the $3^{5}$-groups discussed earlier. Hence, in order to complete the reduction to the $3 B$-pure case, it suffices to find the normalizer of $F$. But now its intersection with $\left(\mathbf{F}_{9}\right)^{2}$ is a $3 B_{4}$-group, and this is determined as the intersection of all the $3 B$-pure $3^{3}$-groups in $F$. 
We have now reduced to the $3 B$-pure case, so we must study the structure of the centralizer of a typical $3 B$-element $t$ in some detail. Here it is necessary to use the notation introduced in [3] to describe the subgroup $3^{1+12} \cdot 2 \mathrm{Suz}$ of the Monster. Briefly, $3^{1+12}$ is written as the central product of 6 copies of $3^{1+2}$, and $2 S u z$ is written as $6 \times 6$ matrices acting on this decomposition. The matrix elements are quaternions reduced modulo 3 , and the vector coordinates (that is, the elements of $3^{1+2}$ modulo the centre) are quaternions reduced modulo $\theta=i+$ $j+k$ (on the left). First we obtain generators for most of the group $C(t)$ by centralizing the $3 C$-element $(i, i, 0,0,0,0) \cdot\langle\omega, \bar{\omega},+, \longrightarrow\rangle$ in the Monster. We obtain $N(3 B) \cong\left(3^{3} \times 3^{1+2}\right) \cdot 3^{1+2}: 2 S_{4}$, where the bracketed normal subgroup $3^{3} \times 3^{1+2}$, which we denote by $T$, is contained in the corresponding group $3^{1+12}$ in the Monster, and may be generated by the elements $(0,0,0,1,1,1),(0,0,0, i, i, i)$, $(1,-1,0,0,0,0),(0,0,1,0,0,0)$ and $(0,0, i, 0,0,0)$. We can extend by an outer automorphism group $3 \times 2 A_{4}$ generated by $\langle 1,1, i, i, i, i\rangle$ and $(-i, i, 0,0,0,0)$. $\langle\omega, \omega, \omega, \omega, \omega, \omega\rangle$ together with the central 3 -element $(i,-i, 0,0,0,0) \cdot d$, where $d$ is the matrix

$$
\left(\begin{array}{cccccc}
1 & \theta & 0 & 0 & 0 & 0 \\
\theta & 1 & 0 & 0 & 0 & 0 \\
0 & 0 & 1 & 1 & 1 & 1 \\
0 & 0 & -1 & -1 & 1 & 1 \\
0 & 0 & -1 & 1 & -1 & 1 \\
0 & 0 & -1 & 1 & 1 & -1
\end{array}\right) .
$$

[Warning: $d$ is an element of the Monster, but not of $T h$.]

First we study the normal subgroup $T$ in some detail. This group $T$ contains four conjugate elementary Abelian $3^{5}$-groups, whose union is the whole group. Now elements of $T h$-classes $3 A, 3 B, 3 C$ are of $M$-classes $3 A, 3 B, 3 B$ respectively. Hence the group generated by $(1,-1,0,0,0,0)$ and $(0,0,1,0,0,0)$ contains two $3 A$-elements and two elements of class $3 B$ or $3 C$, so it is of type $3 A_{2} C_{2}$. This implies that these $3^{5}$-groups are conjugate to $F$, and we can use this to determine the classes of the elements in $T$. We have the following orbits under $2 A_{4}$ :

$$
\begin{array}{ll}
\begin{array}{l}
(1,-1,0,0,0,0),(1,-1,0,1,1,1) \\
(0,0,1,0,0,0),(0,0, \pm 1,1,1,1), \\
*(1,-1,1, i, i, i)
\end{array} & 9 \text { elements of class } 3 A \\
*(0,0,-1, i, i, i),(1,-1, \pm 1,0,0,0), \\
\left.\begin{array}{ll}
(1,-1, \pm 1,1,1,1),(-1,1, \pm 1,1,1,1)
\end{array}\right) & 9 \text { elements of class } 3 A \\
*(0,0,1, i, i, i), *(1,-1,-1, i, i, i) & 9 \text { elements of class } 3 C \\
(0,0,0,1,1,1) & 4 \text { elements of class } 3 B
\end{array}
$$

This gives the conjugacy classes of all the elements in $T$, since multiplying by $t$ does not change the class. Note that the signs in the cases marked ${ }^{*}$ are rather subtle. In order to prove that they are as given, we need to use some later results. However, we do not use these subtleties, so no details are given here. 
Now let us consider the action of the quotient group $3^{1+2}: 2 S_{4}$ on the group $T$. Certainly it fixes the centre, which is an elementary Abelian group of order $3^{4}$, and also fixes the subgroup of index 3 therein consisting of all the $3 B$-elements. Furthermore, the central element of $3^{1+2}: 2 S_{4}$ acts non-trivially on this $3^{4}$, so we have a faithful representation of this group. Since the 2-space stabilizer $3^{4}:\left(2 A_{4}\right.$ $\left.\times 2 A_{4}\right)$ in $S L_{4}(3)$ does not contain a group $3^{1+2}: 2 A_{4}$, it follows that the 4-dimensional module for the latter group is uniserial $1+2+1$. In particular, $N(3 B)$ is transitive on the central $3 A$-elements in $T$, and on the central $3 B$-elements in $T$ outside the derived group $\langle t\rangle$.

Now there are also non-central $3 A$-elements in $T$, and since there are only two classes of $3 A_{3} B_{1}$-groups in $T h$, it follows that $N(3 B)$ is also transitive on these $3 A$-elements. It is clear then that $N(3 B)$ is also transitive on the $3 C$-elements and the non-central $3 B$-elements in $T$.

For the sake of convenience we call the central $3 B^{2}$-group in $T$ a type 1 group, and the non-central one type 2 (both containing the original element $t$ ). It will be clear later that each is the unique $3 B^{2}$-group with the appropriate centralizer order, and therefore the normalizer is in each case transitive on the non-trivial elements. We have $N\left(3 B^{2}\right)_{1} \cong 3^{2} \cdot\left[3^{7}\right] \cdot 2 S_{4}$, which will later turn out to be a maximal subgroup of $T h$, and $N\left(3 B^{2}\right)_{2} \cong 3^{2} \cdot\left[3^{5}\right] .2 S_{4}$, which we proceed to show is contained in $N(3 B)$. Consider the $3 B^{2}$-group which is central in $T$ but does not contain $t$. There is a unique class of such groups, and the normalizer of one of these in $N(t)$ is $\left(3^{3} \times 3^{1+2}\right) \cdot 3.2 S_{4}$. Now this $3 B^{2}$-group is not of type 1 , for if it were then there would be a $3 B$-pure $3^{3}$-group such that all the $3 B^{2}$-subgroups were of type 1 , and hence the normalizer would have to be $3^{3} \cdot 3^{4} \cdot L_{3}(3)$, which is absurd since then $T h$ would have a subgroup $3^{3}: 13 \times 3$. Indeed, this all happens inside the group $F$ described earlier, in which we can see there are just two classes of $3 B^{2}$-groups, one being of type 1 and all the rest of type 2 . Hence $N\left(3 B^{2}\right)_{2}$ is contained in $N(3 B)$, as claimed. Now any larger elementary Abelian $3 B$-pure subgroup of $T$ is again in the $3^{5}$-group, which is conjugate to $F$, so has order $3^{3}$ and contains a unique $3 B^{2}$-group of type 1 . Hence its normalizer is in $N\left(3 B^{2}\right)_{1}$.

Having dealt with all subgroups of $T$, we must next find the conjugacy classes of elements of order 3 in $N(t) /\langle t\rangle$ outside $T /\langle t\rangle$. The quotient group $N(t) / T \cong$ $3^{1+2}: 2 S_{4}$ contains five classes of elements of order 3 , four of which we have nice representatives for:

$\begin{array}{llll}\text { Name } & \text { Representative } & \begin{array}{l}\text { Class } \\ \text { in Suz }\end{array} & \begin{array}{l}\text { Centralizer } \\ \text { in } 3^{1+2}: 2 A_{4}\end{array} \\ 3 a & (i,-i, 0,0,0,0) \cdot d & 3 B & 3^{1+2}: 2 A_{4} \\ 3 b & ? & 3 B & 3^{3} \\ 3 c & (-i, i, 0,0,0,0) \cdot\langle\omega\rangle & 3 A & 3^{2} \times S_{3} \\ 3 d & \langle\omega\rangle \cdot d & 3 B & 3^{2} \times S_{3} \\ 3 e & (-i, i, 0,0,0,0) \cdot\langle\bar{\omega}\rangle \cdot d & 3 B & 3^{2} \times S_{3}\end{array}$


Note: in this table, and below, we write $\langle\omega\rangle$ for the diagonal matrix $\langle\omega, \omega, \omega, \omega, \omega, \omega\rangle$. Each element has an associated vector, which is the vector in $3^{12}$ that we have to divide by in order to get an element in $6 \mathrm{Suz}$-so that for example the vector associated with the $3 a$-element given above is $(i,-i, 0,0,0,0)$.

For each of these five classes we must find the classes they lift to in the non-split extension of the 5-dimensional vector space $T /\langle t\rangle$ by the group $C(t) / T \cong 3^{1+2}: 2 A_{4}$. Now by general principles, an element $x$ is conjugate to its multiples by vectors in the image of $x-1$. Hence we must find the orbits of the centralizer of $x$ in $3^{1+2}: 2 S_{4}$ on the vectors modulo the image of $x-1$. Or to be more precise, we need the orbits on the coset of this generated by the vector associated with $x$.

We deal first with the case $3 a$, and we take the element $(i,-i, 0,0,0,0) \cdot d$, where $d$ is the matrix displayed above. Then the image of $x-1$ is generated by $(0,0,0,1,1,1)$ and $(0,0,0, i, i, i)$, and the vector associated with $x$ is of course $(i,-i, 0,0,0,0)$. Then the group $3^{1+2} 2 A_{4}$ acts on the relevant coset of $T / \operatorname{Im}(x-1)$ with orbits of sizes $3+24$, so we have two conjugacy classes of elements in $C(3 B)$. In order to indentify their classes in $T h$, we first identify their classes in $M$. The given vectors are not in the subspace of $3^{1+12}$ generated by the fixed space of $d$ and its centralizer in $3^{1+12}$. Hence by [3] the elements either have order 9 or are of $M$-class $3 C$. But the latter case cannot happen, so by looking at the centralizer orders of the elements of order 9 we have the two classes:

$\begin{array}{lll}\text { Representative } & \text { Centralizer order } & \text { Type } \\ (i,-i, 0,0,0,0) \cdot d & 2^{3} \cdot 3^{6} & 9 A \\ (i,-i, 1,0,0,0) \cdot d & 3^{6} & 9 B\end{array}$

Next consider the case $3 c$, taking $x$ to be the element $(-i, i, 0,0,0,0) \cdot\langle\omega\rangle$. Then the image of $x-1$ is generated by $(0,0,1,0,0,0)$ and $(0,0,0,1,1,1)$, and the vector associated with $x$ is $(-i, i, 0,0,0,0)$. The quotient of $T$ by the image of $x-1$ may therefore be generated by $(1,-1,0,0,0),(0,0,0, i, i, i)$ and $(0,0, i, 0,0,0)$. If we add a vector in the space generated by the first two vectors, then we get an element of order 9, see [3]. Hence all these elements are of class $9 C$ and are conjugate. Then the $3 c$-centralizer in $3^{1+2} 2 S_{4}$ has 3 orbits on the remaining vectors in the coset, and two of these orbits are interchanged by the outer automorphism. In all 3 cases the corresponding $\left[3^{2}\right]$-group has type $3 B_{4}$ in the Monster, and centralizes an element of $T h$-class $3 C$ in $T$, so from what we know about the $3 C$-centralizer we can deduce that the whole centralizer is 
conjugate to $E$. Thus we have the 3 classes:

$\begin{array}{lll}\text { Representative } & \text { Centralizer } & \text { Type } \\ (-i, i, 0,0,0,0)\langle\omega\rangle & 9 \times 3^{2}: 2 & 9 C \\ (-i, i, i, 0,0,0)\langle\omega\rangle & 3^{5} & 3 B_{4} \\ (-j, j, i, 0,0,0)\langle\omega\rangle & 3^{5} & 3 B_{1} C_{3}\end{array}$

Let us turn now to the case $3 d$, taking $x$ to be the element $\langle\omega\rangle d$. In this case the image of $x-1$ is generated by $(0,0,0,1,1,1)$ and $(0,0,-1, i, i, i)$, and does not contain our original $3 B$-element $t$. Also, the vector associated with $x$ is $(0,0,0,0,0,0)$. Note that this implies that elements are not necessarily conjugate to their multiples by $t$. We must determine the orbits of $C(x)$ on the vectors of the space generated by $(1,-1,0,0,0,0),(0,0,1, i, i, i)$ and $(0,0, i, 0,0,0)$, say. If we ever add in the last generator, then the element has order 9 modulo $\langle t\rangle$, so we can neglect this case. Now $x$ itself gives rise to a $3^{2}$-group of $M$-type $3 A_{1} B_{3}$, so of Th-type $3 A_{1} B_{2} C_{1}$. But the centralizer of such a $3^{2}$-group is just $3^{5}: 2$, so by counting we see that all $3^{2}$-groups of type $3 d$ are conjugate to it. We therefore have only one class of $3^{2}$-group as follows:

$\begin{array}{lll}\text { Representative } & \text { Centralizer } & \text { Type } \\ \langle\omega\rangle \cdot d & 3^{5}: 2 & 3 A_{1} B_{2} C_{1}\end{array}$

Next we turn to the $3 e$ case, taking $x$ to be the element $(-i, i .0,0,0,0) \cdot\langle\bar{\omega}\rangle \cdot d$, so that the fixed space of $x$ is generated by $(0,0,0,1,1,1)$ and $(0,0,1, i, i, i)$, and the vector associated with $x$ is $(-i, i, 0,0,0,0)$. We may suppose that the added vector is in the space generated by $(1,-1,0,0,0,0),(0,0,-1, i, i, i)$, and $(0,0, i, 0,0,0)$, say. If ever we add in the last generator, then the resulting element has order 9 modulo $\langle t\rangle$, so we can neglect it. If we add in only $(1,-1,0,0,0,0)$, then the resulting $3^{2}$-group is still in the involution centralizer, so the structure of $2^{1+8} \cdot A_{9}$ implies that it is conjugate to the first $3^{2}$-group $\langle t, x\rangle$. And finally, the element $(0,0,-1, i, i, i)$ is in the fixed space of $x$, so gives rise to a $3^{2}$-group with the same centralizer. We have the two cases:

$\begin{array}{lll}\text { Representative } & \text { Centralizer } & \text { Type } \\ (-i, i, 0,0,0,0) \cdot\langle\bar{\omega}\rangle \cdot d & 3^{5}: 2 & 3 B_{4} \\ (-i, i, 1, i, i, i) \cdot\langle\bar{\omega}\rangle \cdot d & 3^{5} & 3 B_{1} C_{3}\end{array}$

In here we identify the conjugacy classes by observing that $E$ must be a subgroup of $C(3 B)$, and can intersect $T$ in at most a $3^{3}$-subgroup. Hence it maps onto a $3^{2}$-subgroup of $3^{1+2}: 2 S_{4}$, containing no $3 a$-elements or $3 d$-elements, so containing $3 b, 3 c$ and $3 e$-elements. In fact this $3^{2}$-group has type $3 b_{1} c_{2} e_{1}$. 
Before considering the $3 b$-case, which is the only one left, let us find the normalizers of everything not involving the $3 b$-cosets. We can ignore the $3 a$-elements, since they lift only to elements of order 9. If we have a $3 c, 3 d$ or $3 e$-element, then the centralizer is an elementary Abelian $3^{5}$-group containing a $3 C$-element, so its normalizer has already been found. Hence we can restrict to the case when we have only a $3 b$-element outside $T$.

Here we are somewhat hampered by not having an explicit element to work with, so we have to use a rather clumsy theoretical argument to classify the conjugacy classes.

Now we have already shown, in the discussion of the structure of $N(3 B)$, that a $3 b$-element centralizes a $3^{3}$-subgroup of $T$, and we know what this is by looking inside $F$. Indeed, it has type $3 B_{4} C_{9}$, and so may be taken to be generated by $(0,0,0,1,1,1)$ and $(1,-1,1,0,0,0)$. Now if we multiply by any element of the central $3^{4}$ of $T$ then the resulting element still centralizes a $3 C$-element, so has order 3 and is in one of the $3^{5}$-groups already considered. Finally we wish to prove that if we multiply by any other element of $T$ then we get an element of order 9 . Now $T / \operatorname{Im}(x-1) \cong 3^{3}$ so there are 27 cosets to consider, of which we have dealt with 9 . But now $C(9 A) \cong 3^{3} \cdot 3^{1+2}: Q_{8}$ regarded as an element of type $3 a$ (that is, if it cubes to $t$ then the normal $3^{3}$ is the intersection with $T$ ). Hence there exist elements of order 9 of type $3 b$, for otherwise we could multiply a $3 b$-type element of order 3 by a commuting $9 A$-element to obtain a $3 b$-type element of order 9 . Hence all the remaining elements have order 9 , as we have already seen that multiplying by a central element of $T$ does not affect the order of the element modulo $\langle t\rangle$. (This is true for elements of order 3 , so it is also true for elements of order 9 , since $T /\langle t\rangle$ is Abelian.) Now these 9-elements do not cube to $t$, so there are just three classes of elements of order 3 modulo $\langle t\rangle$, each with centralizer $3^{5}$, the corresponding $3^{2}$-groups being one of type $3 B_{4}$ and two of type $3 B_{1} C_{3}$. Finally we notice that in this case also the centralizer of such an outer element of order 3 is just the group $E \cong 3^{5}$, whose normalizer we have already found.

This concludes the proof of

THEOREM 4.1. Any 3-local subgroup of Th is contained in one of the following maximal 3-local subgroups

$$
\begin{aligned}
& N(3 A) \cong\left(3 \times G_{2}(3)\right): 2, \\
& N(3 B) \cong\left(3^{3} \times 3_{+}^{1+2}\right) \cdot 3_{+}^{1+2}: 2 S_{4}, \\
& N\left(3 B^{2}\right) \cong 3^{2} \cdot\left[3^{7}\right] \cdot 2 S_{4}, \\
& N(3 C) \cong\left(3 \times 3^{4}: 2 A_{6}\right): 2 .
\end{aligned}
$$




\section{Non-local subgroups}

Using the classification of finite simple groups, we can find all non-Abelian simple groups whose order divides that of the Thompson group, $|T h|=$ $2^{15} \cdot 3^{10} \cdot 5^{3} \cdot 7^{2} \cdot 13 \cdot 19.31$. We divide these into two cases:

(1) Known or possible subgroups:

$A_{5}, A_{6}, L_{2}(7), L_{2}(8), L_{2}(13), L_{2}(19)$ ?, $L_{3}(3), U_{3}(3), U_{3}(8), G_{2}(3)$, and ${ }^{3} D_{4}(2)$.

(2) Non-subgroups:

$A_{7}, A_{8}, A_{9}, A_{10}, L_{2}(25), L_{2}(27), L_{2}(31), L_{2}(49), L_{2}(64), L_{2}(125), U_{3}(4)$, $U_{3}(5), L_{3}(4), L_{3}(5), L_{3}(9), U_{4}(2), U_{4}(3), L_{4}(3), L_{5}(2), L_{6}(2), S_{6}(2), O_{8}^{+}(2), S_{4}(8)$, $S_{6}(3), O_{7}(3), S z(8),{ }^{2} F_{4}(2)^{\prime}, G_{2}(4)$ and $J_{2}$.

We prove that none of the groups listed in (2) is in Th. First note that it suffices to prove it for $A_{7}, L_{3}(5), L_{2}(25), L_{2}(27), L_{3}(4), S z(8), L_{2}(31),{ }^{2} F_{4}(2)^{\prime}, L_{2}(49)$, $L_{2}(64), L_{2}(125), L_{3}(9), U_{3}(4), J_{2}$, and $U_{4}(2)$.

Now the 3-elements in any $A_{5}$ are of class $3 B$ (see Proposition 5.1 below), and $C(3 B)$ does not contain $A_{4}$, so there is no $A_{7}$. Similarly, the 3-elements in $31: 3$ are of class $3 C$, so there is no $L_{3}(5)$. For $L_{2}(25)$, note that the 4-elements in $S_{5}$ are of class $4 B$ (see below), but there is no class of 12-elements in $T h$ which powers to both $3 B$-elements and $4 B$-elements.

We eliminate the groups $L_{2}(31), L_{2}(49), L_{2}(64), L_{2}(125), L_{3}(9)$ and ${ }^{2} F_{4}(2)^{\prime}$ since they contain elements of orders $16,25,63,63,91$ and 16 respectively. Similarly, $U_{3}(4)$ and $J_{2}$ contain $5 \times A_{5}$ and $L_{2}(27)$ contains $3^{3}: 13$, while $L_{3}(4)$ and $U_{4}(2)$ contain subgroups of the shape $2^{4}: A_{5}$. In each case we know from the local analysis that $T h$ does not contain such a group. Finally, it is easy to show that there is no restriction of the character of degree 248 to $S z(8)$.

Conversely, J. G. Thompson has shown that $T h$ contains subgroups of the shapes $U_{3}(8): 6$ and ${ }^{3} D_{4}(2): 3$, by looking inside the Monster, and S. P. Norton has shown similarly that $T h$ contains $M_{10} \cong A_{6} \cdot 2$ (see Proposition 5.8 below). Then the $3 A$-centralizer contains $G_{2}(3)$, which contains all the remaining groups on the list except for $L_{2}(19)$. I do not yet know whether $L_{2}(19)$ is a subgroup of Th.

In what follows, we make considerable use of structure constants. If $X, Y$, and $Z$ are three conjugacy classes in $G$, then $\xi_{G}(X, Y, Z)$ denotes the value of the expression

$$
\frac{|G|}{|C(x)||C(y)||C(z)|} \sum \frac{\chi(x) \cdot \chi(y) \cdot \chi(z)}{\chi(1)}
$$

where $x \in X, y \in Y, z \in Z$, and the sum is over all irreducible characters $\chi$ of $G$. It is a well-known fact that

$$
\xi_{G}(X, Y, Z)=\sum \frac{1}{|C(x, y, z)|}
$$


where the sum is over all conjugacy classes of triples $(x, y, z)$ of such elements with $x y z=1$.

Proposition 5.1. There is a unique class of $A_{5}$ in $T h$, and it has normalizer $S_{5}$.

Proof. The only non-zero structure constant of type $(2,3,5)$ is $\xi(2 A, 3 B, 5 A)=$ 1. But there is no $A_{5}$ in any of the element centralizers.

Proposition 5.2. (a) There is a unique class of $L_{2}(8)$, and its normalizer is $S_{3} \times L_{2}(8): 3$, which is contained in $N(3 A) \cong\left(3 \times G_{2}(3)\right): 2$.

(b) There is a unique class of ${ }^{3} D_{4}(2)$, and its normalizer is ${ }^{3} D_{4}(2): 3$.

Proof. The elements of order 3 in $L_{2}(8)$ are cubes, so are of class $3 B$. Now the 248-character restricts to ${ }^{3} D_{4}(2)$ as the direct sum of the irreducible representations of degrees 52 and 196, so in particular the elements of ${ }^{3} D_{4}(2)$-class $3 B$ are of $T h$-class $3 B$. Then the structure constant $\xi_{T h}(2 A, 3 B, 7 A)=7 / 6$ is entirely accounted for by the contributions from the known classes of $L_{2}(8)$ and ${ }^{3} D_{4}(2)$, since $\xi_{L_{2}(8)}(2,3,7)=3$ and in ${ }^{3} D_{4}(2)$ we have $\xi(2 B, 3 B, 7 D)=3$.

REMARK. It is possible to give an alternative proof of the uniqueness of ${ }^{3} D_{4}(2)$ as a subgroup of $T h$, by constructing the group out of its 7-local subgroups.

PROPOSITION 5.3. There is a unique class of $U_{3}(8)$ in Th, and its normalizer is $U_{3}(8): 6$.

Proof. Any group $U_{3}(8)$ may be constructed by taking a group $3 \times L_{2}(8)$, and extending the $2^{3}$-normalizer from $3 \times 2^{3}: 7$ to $2^{3+6}:(7 \times 3)$. Now there is a unique class of $3 \times L_{2}(8)$ in $T h$, which is contained in $3 \times G_{2}(3)$, and the entire $2^{3}$-normalizer in $T h$ has the shape $2^{3} \cdot\left[2^{8}\right] \cdot\left(S_{3} \times L_{2}(7)\right)$. But in the latter group the elements of order 21 act on the $\left[2^{8}\right]$-factor as the direct sum of irreducible representations of degrees 6 and 2 . Hence there is a unique group $2^{3+6}: 21$ containing a given $2^{3}: 21$, and the result follows.

Proposition 5.4. There is a unique class of $L_{2}(13)$ in $T h$, and its normalizer is $\left(3 \times L_{2}(13)\right): 2$, which is contained in $N(3 A) \cong\left(3 \times G_{2}(3)\right): 2$.

Proof. Since the total $(2,3,7)$-structure constant in $L_{2}(13)$ is 6 , and the centralizer of any $L_{2}(13)$ in $T h$ has order at most 3 , it follows that any $L_{2}(13)$ contributes at least 1 to the $(2,3,7)$-structure constant in $T h$. But $\xi(2 A, 3 A, 7 A)=$ $3 / 14$, and we have already accounted for all of $\xi(2 A, 3 B, 7 A)=7 / 6$, so the class 
fusion must be $(2 A, 3 C, 6 A, 7 A, 13 A)$. Now the only $L_{2}(13)$ with non-trivial centralizer is the one with normalizer $\left(3 \times L_{2}(13)\right): 2$, contained in $\left(3 \times G_{2}(3)\right): 2$, and this $L_{2}(13)$ extends to $L_{2}(13): 2$. Furthermore, $\xi(2 A, 3 C, 7 A)=4$, so any $L_{2}(13)$ with trivial centralizer also extends to $L_{2}(13): 2$. If we restrict the 248-dimensional representation to each of the groups $13: 12, L_{2}(13): 2$ and $\left(3 \times G_{2}(3)\right): 2$ in turn, we find that each of these groups fixes a unique 1-space pointwise. Thus any $L_{2}(13)$ is contained in $G_{2}(3)$, and the result follows.

Proposition 5.5. There is a unique class of $G_{2}(3)$ in Th, and its normalizer is $\left(3 \times G_{2}(3)\right): 2$.

Proof. Any group $G_{2}(3)$ may be constructed from $L_{2}(13)$ by extending $D_{14}$ to 7:6. But both the $L_{2}(13)$-normalizer $\left(3 \times L_{2}(13)\right): 2$ and the $D_{14}$-normalizer $7: 6 \times S_{3}$ are contained in $\left(3 \times G_{2}(3)\right): 2$, and the result follows.

Proposition 5.6. If $L_{2}(19)$ is a subgroup of $T h$, then there is exactly one class, and its normalizer is $L_{2}(19): 2$.

Proof. The 248-character restricts to $U_{3}(8)$ as $1 a+57 a b+133 a$, so the 9-elements in $19: 9$ are $9 C$-elements. Thus any $L_{2}(19)$ has type $(2 A, 3 B, 5 A, 9 C, 10 A, 19 A)$. Now $\xi_{T h}(2 A, 3 B, 9 C)=6$, of which an amount $1 / 6$ is attributable to $L_{2}(8)$. But the total $(2,3,9)$-structure constant in $L_{2}(19)$ is 6 , and the result follows from the fact that the elements of order 19 in $T h$ are self-centralizing.

We conclude with a few remarks about subgroups isomorphic to $A_{6}, L_{2}(7)$, $L_{3}(3)$ and $U_{3}(3)$.

Proposition 5.7. Any $A_{6}$ in $T h$ is of type $(2 A, 3 B, 3 B, 4 B, 5 A)$. Hence the degree 248 character of Th restricts to any $A_{6}$ as $5 a^{3} b^{3}+8 a^{4} b^{4}+9 a^{6}+10 a^{10}$.

Proof. Firstly, it contains $3 B$-elements since it contains $A_{5}$. Secondly, it contains $4 B$-elements since it has trivial centralizer and $\xi(2 A, 4 A, 5 A)=1 / 4$.

REMARK. This proof also shows that the $S_{5}$ contains $4 B$-elements.

Proposition 5.8. There exists a subgroup $A_{6}$ with normalizer $M_{10}$. This group $A_{6}$ together with $S_{5}$ generate Th.

Proof. In the Monster there is a group $\left(A_{6} \times A_{6} \times A_{6}\right) \cdot\left(2 \times S_{4}\right)$. Centralizing a $3 C$-element permuting the three factors of the minimal normal subgroup of this, we have a group $M_{10}$. Now using the " $Y$ "-generators for $M$ (see [1], page 
232), this $3 C$-element rotates the three arms of the $Y$, giving a subgroup of $T h$ as a quotient of the inner half of the infinite Coxeter group with diagram

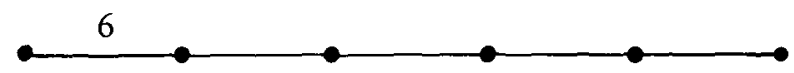

By covering up each of the nodes in turn we obtain the subgroups $A_{6}, S_{5}$, $\left(6 \times A_{4}\right): 2, \ldots$ of $T h$. Now the first two of these groups intersect in $A_{5}$, and generate the third, which does not normalize the $A_{6}$ and so extends it to $T h$.

Proposition 5.9. If there is a subgroup $S_{6}$ in Th, then there is a unique class.

Proof. Any group $S_{6}$ can be constructed by taking $S_{5}$ and adjoining an involution commuting with a subgroup $S_{4}$. But the $A_{4}$-normalizer is $\left(A_{4} \times\right.$ $\left.2 A_{4}\right): 2$, so there is a unique way of making this extension.

Remark. S. P. Norton has shown that $S_{6}$ is not a subgroup of $T h$, as follows. Let $a, b, c, d, e, f$ be generators of Th wr 2 corresponding to the nodes of the above Coxeter graph in order. Then the putative $S_{6}$ in $T h$ would be generated by $\left\{(a b)^{3}, a c, a d, a e, a f\right\}$, and in particular would contain the element $(a b)^{3} a c$. But we can calculate the order of this element in the Monster, since it is contained in a known subgroup $O_{7}(3)$. It turns out to have order 9 , which is a contradiction.

Proposition 5.10. (a) There is a unique class of $L_{2}(7)$ containing 3 A-elements, and its normalizer is $\left(L_{2}(7) \times 7: 3\right): 2$, which is contained in ${ }^{3} D_{4}(2): 3$.

(b) There is no $L_{2}(7)$ containing $3 B$-elements.

Proof. (a) The structure constant $\xi(2 A, 3 A, 7 A)=3 / 14$ is completely accounted for by the contributions $1 / 21$ from $\left(L_{2}(7) \times 7: 3\right): 2$ inside ${ }^{3} D_{4}(2): 3$ and $1 / 6$ from $S_{4} \times 2^{3} \cdot L_{3}(2)$ inside $2^{5} \cdot L_{5}(2)$.

(b) The structure constant $\xi(2 A, 3 B, 7 A)=7 / 6$ has already been fully accounted for by $L_{2}(8)$ and ${ }^{3} D_{4}(2): 3$ (see Proposition 5.2).

REMARK. $\xi(2 A, 3 C, 7 A)=4$, of which an amount 1 has already been accounted for by $L_{2}(13)$.

Proposition 5.11. There is a unique class of $U_{3}(3)$ whose non-3-central 3-elements are of class $3 A$, and its normalizer is $3 \times U_{3}(3): 2$, which is contained in $\left(3 \times G_{2}(3)\right): 2$. 
Proof. Any group $U_{3}(3)$ can be constructed by taking a group $L_{2}(7)$ and extending a subgroup $S_{3}$ to $S_{3} \times 3$. But by Proposition 5.10 any $L_{2}(7)$ containing $3 A$-elements has normalizer contained in ${ }^{3} D_{4}(2): 3$. Furthermore the $S_{3}$ has normalizer $S_{3} \times L_{2}(8): 3$, which is contained in the same group ${ }^{3} D_{4}(2): 3$. Since there is a unique class of $U_{3}(3)$ in ${ }^{3} D_{4}(2)$, the result follows.

Proposition 5.12. Any other $U_{3}(3)$ in Th has type $(2 A, 3 B, 3 C, 4 A, 4 A, 6 C, 7 A$, $8 A, 12 C)$.

Proof. In any $U_{3}(3)$ the 3-central 3-elements have centralizer $3^{1+2}: 4$, so are of class $3 B$. The remaining 3 -elements are contained in $L_{2}(7)$, so are of class $3 C$, since we have excluded the $3 A$-case. The only difficulty now is to identify the second class of elements of order 4 , but only $4 A$ gives integral trace on restricting the 248-character.

In the case of $L_{3}(3)$, we have very little information. The 3-central 3-elements have centralizer $3^{1+2}: 2$, so again are of class $3 B$. The remaining 3-elements are of class $3 B$ or $3 C$, since they normalize elements of order 13 . Furthermore, they are contained in $S_{4}$, so if they are $3 B$-elements then the 4-elements are of class $4 B$, since $\xi(2 A, 3 B, 4 A)=0$.

REMARK. It may well be possible to complete the enumeration of the maximal subgroups of $T h$ by computer. The first, and perhaps biggest, problem is to reconstruct $T h$ as a group of 248 by 248 matrices, preferably over $F_{2}$ for efficiency of calculation. Then the enumeration of $L_{2}(7)$ and $U_{3}(3)$ is almost algorithmic, by taking 7:3 and extending the 3-normalizer. Similarly, the cases $L_{3}(3)$ and $L_{2}(19)$ could probably be dealt with by taking $13: 3$ or $19: 3$ and again extending the 3-normalizer. The case of $A_{6}$ seems rather harder, but can perhaps be approached via the 2-local subgroups.

\section{Acknowledgements}

I would like to thank Dr. D. C. Hunt and the University of New South Wales for their hospitality and a Visiting Fellowship in November and December 1983, during which time I began the work on 3-local subgroups which occupies the bulk of this paper. 


\section{References}

[1] J. H. Conway, R. T. Curtis, S. P. Norton, R. A. Parker and R. A. Wilson, An ATLAS of finite groups, (Oxford Univ. Press, 1985).

[2] P. E. Smith, On certain finite simple groups, (Ph.D. thesis, Cambridge, 1975).

[3] R. A. Wilson, 'The odd-local subgroups of the Monster', J. Austral. Math. Soc. 44 (1988), 1-16.

Department of Pure Mathematics and Mathematical Statistics

University of Cambridge

16 Mill Lane

Cambridge CB2 1SB

England 